\title{
Protective Effect of Ischemic Preconditioning against Liver Injury after Major Hepatectomy Using the Intermittent Pringle Maneuver in Swine
}

\author{
Mitsugi Shimoda Yoshimi Iwasaki Tokihiko Sawada Keiichi Kubota \\ Department of Gastroenterological Surgery, Dokkyo University School of Medicine, Mibu, Japan
}

\section{Key Words}

Reperfusion injury • Ischemic preconditioning •

Intermittent clamping

\begin{abstract}
Objective: To investigate whether ischemic preconditioning (IP) protects the liver against ischemia-reperfusion injury (I/ $\mathrm{R}$-I) after major hepatectomy through intermittent hepatic pedicle clamping (IC) in a swine liver resection model. Background: Although many studies have reported a protective effect of IP against continuous hepatic ischemia, it has not been elucidated whether IP protects the liver against I/R-I after hepatectomy using IC. This is the first study to evaluate the effect of IP in a swine major hepatectomy model using IC. Methods: Pigs $(n=12)$ were divided into 2 groups (IP or non-IP). In the IP group, livers were subjected to IP (10 min ischemia and 10 min reperfusion) before liver resection using IC (15 min ischemia and 5 min reperfusion). A left hemihepatectomy was then performed using IC in both groups. Hemodynamic changes and plasma concentrations of aspartate aminotransferase, lactate dehydrogenase, lactic acid and hyaluronic acid were measured at 60, 120 and $180 \mathrm{~min}$ after hepatectomy. Apoptosis (TUNEL staining and electron microscopy), plasma tumor necrosis factor- $\alpha$ (TNF- $\alpha$ ) and $\mathrm{NO}_{2}^{-} / \mathrm{NO}_{3}^{-}$were evaluated for $180 \mathrm{~min}$ after hepatectomy. Results: There were no significant differences in body weight, blood loss, resected liver weight, Pringle time or hemodynamic changes between the 2 groups. IP significantly
\end{abstract}

(c) 2007 S. Karger AG, Basel

1015-2008/07/0741-0042\$23.50/0

Fax +41 613061234 E-Mail karger@karger.ch www.karger.com
Accessible online at: www.karger.com/pat reduced plasma aspartate aminotransferase levels for 180 min after hepatectomy (IP: $135.8 \pm 13.5$ vs. non-IP: $199 \pm$ $16.8 \mathrm{IU} / \mathrm{I} ; \mathrm{p}=0.018$ ). In the non-IP group, apoptotic changes in sinusoidal endothelial cells were observed with increased plasma TNF- $\alpha$ levels. IP protected liver injury from increase in plasma TNF- $\alpha(p=0.042)$. Significantly fewer apoptotic cells were seen in the IP than in the non-IP group $(p=0.002)$. Plasma levels of lactate dehydrogenase, lactic acid and $\mathrm{NO}_{2}^{-} /$ $\mathrm{NO}_{3}^{-}$in the IP group tended to be lower than those in the non-IP group. Conclusions: IP prior to hepatectomy with IC resulted in less hepatic injury and apoptotic cell death than in livers not subjected to IP. IP with IC has the potential to improve the clinical postoperative course of patients undergoing hepatectomy.

Copyright $\odot 2007$ S. Karger AG, Basel

\section{Introduction}

The Pringle maneuver, involving compression of the hepatoduodenal ligament, interrupts most of the blood flow to the liver, but produces profound hepatic ischemia and intestinal congestion unless the clamping is frequently released. Since this maneuver was first reported by Pringle [1] in 1908, it has been used clinically during hepatectomy to reduce blood loss. However, ischemia-reperfusion injury (I/R-I) resulting from the Pringle maneuver is one of the pathogenetic factors involved in postoperative liver dysfunction and hepatic failure, especially 
when the liver is steatotic and cirrhotic [2,3]. Liver resection is widely performed for hepatocellular carcinoma in patients with chronic liver disease due to hepatitis $\mathrm{C}$ or hepatitis B [4-7]. Since Makuuchi et al. [8], who were the first to advocate the usefulness of the hemihepatic pedicle clamping during hepatectomy for diseased liver, various modified Pringle maneuvers such as hemihepatic or intermittent hepatic pedicle clamping (IC) have been tried as candidates for an optimal treatment for deranged liver patients. The IC procedure has been accepted in both experimental and clinical settings [2,9].

Murry et al. [10] were the first to report that a short period of ischemia prior to prolonged ischemia reduced the size of the subsequent myocardial infarct. This phenomenon, known as ischemic preconditioning (IP), has also been reported to protect the liver in patients undergoing hepatectomy. In a rat model, we have already demonstrated that the protective effect of IP was greater against damage induced by intermittent ischemia than that induced by continuous ischemia [11]. However, it is still unknown whether IP might not only reduce I/R-I after continuous but also after intermittent hepatic ischemia in a liver resection model.

If further ischemic tolerance could be promoted by performing IP before IC, this would be more advantageous for clinical surgery, especially in patients with diseased liver. Therefore, we evaluated the effect of IP in a swine hepatectomy model by monitoring hemodynamic and biochemical parameters as well as histological examinations.

\section{Methods}

Male white pigs were used in this study, which was performed in accordance with the Guidelines for the Care and Use of Laboratory Animals of the Dokkyo University School of Medicine. The pigs were allowed free access to water and standard foods before undergoing surgical procedures.

\section{Surgical Procedures}

A chevron incision was performed under general anesthesia. On preliminary examination, we first performed occlusion of the portal vein and hepatic artery without a veno-veno bypass, but all pigs adopted severe hypotension due to intestinal congestion within 5 min after pedicle clamping, and we could not confirm any hepatic resection in this condition. In humans, collateral vessels develop between the portal and systemic vein, and hepatectomy using hepatic pedicle clamping can be safely performed without intestinal congestion and severe hypotension. In pigs, these collateral vessels are very poor; therefore, to prevent splenic and intestinal congestion during prolonged occlusion of the portal vein and hepatic artery, a veno-veno bypass was created between the main splenic vein and the right internal jugular vein by using a venous cannula (V122-16; Stöckert, Munich, Germany) [12]. The hepatic pedicle and all hepatic ligaments were isolated, and total liver ischemia was performed by clamping the hepatic pedicle using a vessel tape. During clamping, a veno-veno bypass was used for preventing bowel congestion and closed during declamping. After clamping the hepatic pedicle, a left hemihepatectomy was performed using IC (cycles of $15 \mathrm{~min}$ ischemia and $5 \mathrm{~min}$ reperfusion). Liver transection was achieved by the crush-clamping method using Pean forceps. During liver transection, each of the exposed Glisson's vessels was ligated with 2-0 or 3-0 silk. Immediately after hepatectomy, the hepatic pedicle was declamped and complete hemostasis was confirmed by suture closure.

During this procedure, hemodynamic parameters (systolic and diastolic arterial pressure, heart rate) were monitored by an arterial line, and intraoperative blood loss was measured. All pigs received Ringer solution during this procedure.

\section{Experimental Groups}

The pigs were divided into 2 groups: (1) an IP group ( $n=6$; IP was performed before hepatectomy using IC) and (2) a non-IP group ( $n=6$; hepatectomy was performed using IC without prior IP). IP was defined as total hepatic ischemia for $10 \mathrm{~min}$ followed by reperfusion for $10 \mathrm{~min}$ prior to hepatectomy.

\section{Measurements and Sampling Protocol}

Blood samples were obtained from the arterial line immediately after laparotomy, after hepatectomy, and at 60, 120 and 180 min after reperfusion. We evaluated serum aspartate aminotransferase (AST), lactate dehydrogenase (LDH), lactic acid (LA), hyaluronic acid (HA), tumor necrosis factor- $\alpha$ (TNF- $\alpha$ ) and $\mathrm{NO}_{2}^{-} / \mathrm{NO}_{3}^{-}$. Hepatic tissues were obtained from the right lobe after reperfusion at $180 \mathrm{~min}$ to evaluate histological findings.

Serum AST, LDH, LA and HA were measured using standard clinical methods for automated analysis (Model 7170; Hitachi Inc., Tokyo, Japan). Plasma TNF- $\alpha$ levels were examined by enzyme-linked immunosorbent assay using a commercial porcine TNF- $\alpha /$ TNFSF2 immunoassay kit (R\&D Systems Inc., Minneapolis, Minn., USA). Plasma $\mathrm{NO}_{2}^{-} / \mathrm{NO}_{3}^{-}$levels were determined by a commercial nitrate/nitrite colorimetric assay kit (Cayman Chemical Company, Ann Arbor, Mich., USA).

\section{Histological Examination}

Tissue samples were obtained from the remnant liver $180 \mathrm{~min}$ after hepatectomy, fixed with $10 \%$ formalin for $24 \mathrm{~h}$, and embedded in paraffin. Three-micrometer-thick sections were stained with hematoxylin and eosin and analyzed by the in situ terminal deoxynucleotidyl transferase-mediated dUTP-biotin nick end labeling (TUNEL) method using an apoptosis in situ detection kit (Wako Pure Chemical Inc., Osaka, Japan) according to the manufacturer's instructions. The mean numbers of apoptotic cells per 10 highpower fields were calculated for the 2 groups and then compared.

Immediately after removal, other hepatic tissues were fixed with $2 \%$ glutaraldehyde in $0.05 \mathrm{M}$ cacodylate buffer $(\mathrm{pH} 7.3)$ for $1.5 \mathrm{~h}$ at $4^{\circ} \mathrm{C}$, postfixed with $\mathrm{OsO}_{4}$ in $0.15 \mathrm{M}$ phosphate buffer $(\mathrm{pH}$ 7.3) for $1.5 \mathrm{~h}$ at $4^{\circ} \mathrm{C}$, dehydrated, and embedded in Epon 812 resin. Ultrathin sections were cut using a Super Nova ultramicrotome (Reichert-Jung, Vienna, Austria), double-stained with uranyl acetate and lead citrate, and examined with a JEM-1210 electron microscope (JEOL, Tokyo, Japan). 


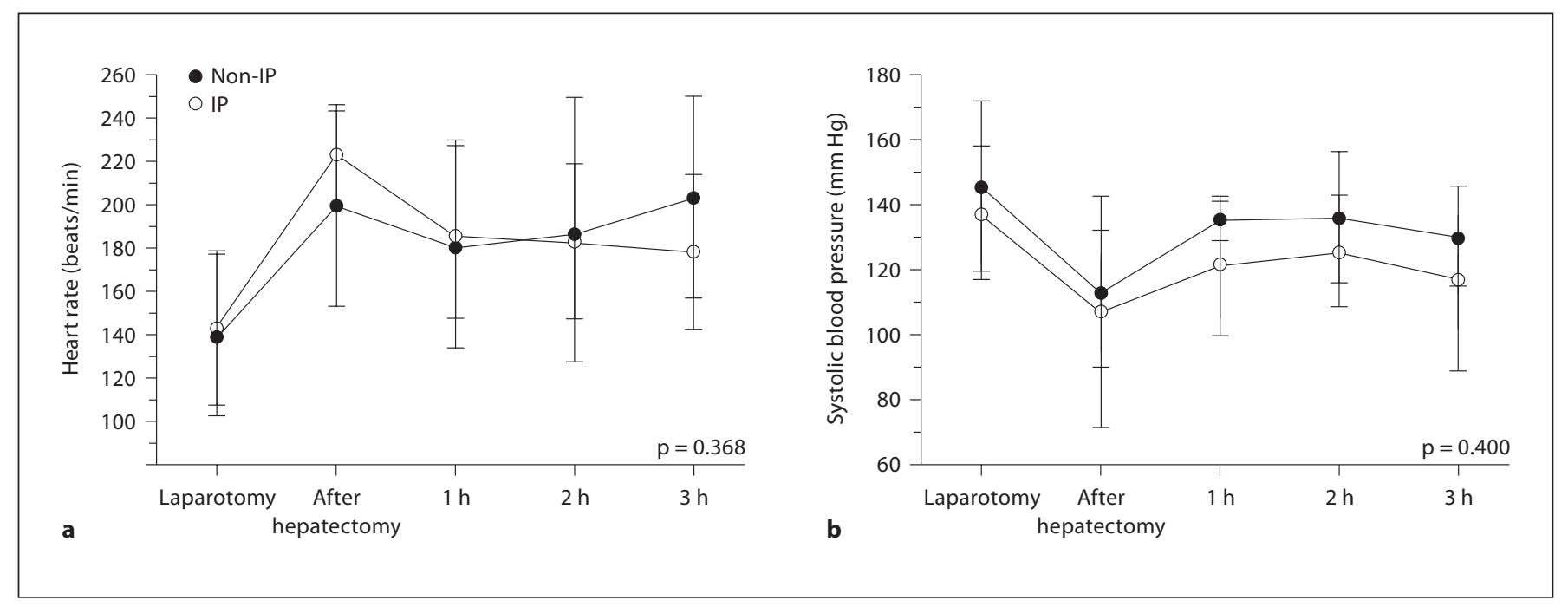

Fig. 1. Heart rate (a) and blood pressure (b) revealed no significant differences between the IP and non-IP group at $3 \mathrm{~h}$.

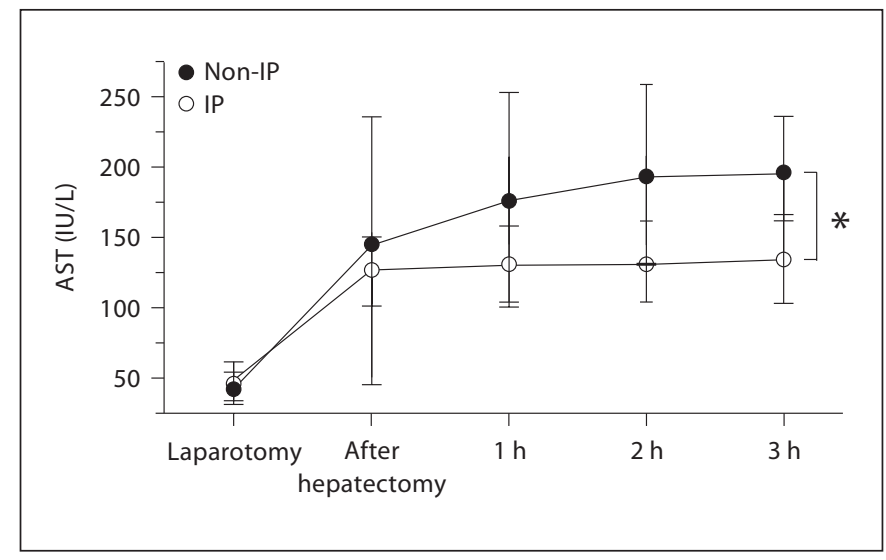

Fig. 2. The serum AST levels in both groups increased gradually during the experimental period and significant differences were observed at $3 \mathrm{~h}$ after reperfusion. ${ }^{*} \mathrm{p}=0.018$.

Table 1. Characteristics of pigs undergoing hepatectomy with IC and using IP

\begin{tabular}{lcl}
\hline & $\begin{array}{l}\text { Non-IP } \\
(\mathrm{n}=6)\end{array}$ & $\begin{array}{l}\text { IP } \\
(\mathrm{n}=6)\end{array}$ \\
\hline BW, kg & $24.2 \pm 3.11$ & $24.8 \pm 2.28$ \\
Blood loss, ml & $57.5 \pm 45.74$ & $60.0 \pm 35.59$ \\
IC time, min & $30.6 \pm 1.78$ & $33.25 \pm 2.87$ \\
RLW, g & $259.2 \pm 49.99(40.7 \%)$ & $288.0 \pm 55.08(45.6 \%)$ \\
\hline
\end{tabular}

BW = Body weight; IC = intermittent hepatic pedicle clamping; RLW = resected liver weight.

\section{Statistical Analysis}

All values are expressed as means $\pm S D$. All parameters were evaluated using Student's t test or the $\chi^{2}$ test. Differences between the 2 groups were evaluated using analysis of variance and considered significant at $\mathrm{p}<0.05$.

\section{Results}

There were no significant differences in body weight, IC time, blood loss or resected liver weight between the IP and non-IP groups (table 1).

\section{Effect of IP on Hemodynamics}

Heart rate was higher immediately after hepatectomy than during the period of laparotomy, and then decreased gradually. There were no significant differences in heart rate between the 2 groups (fig. 1a). Systolic blood pressure was reduced after hepatectomy and was then maintained at the same level thereafter, there being no significant differences between the 2 groups (fig. 1b).

\section{Effect of IP on Serum AST, LDH, LA and HA Levels}

The serum AST levels in both groups increased gradually during the experimental period and significant differences were observed at $180 \mathrm{~min}$ after reperfusion (IP: $135.8 \pm 13.5$ vs. non-IP: $199 \pm 16.8 \mathrm{IU} / \mathrm{l} ; \mathrm{p}=0.018$ ). At other observation points, the AST level in the IP group tended to be lower than that in the non-IP group (fig. 2). The serum LDH levels in the IP group were maintained after reperfusion, whereas those of the non-IP group in- 


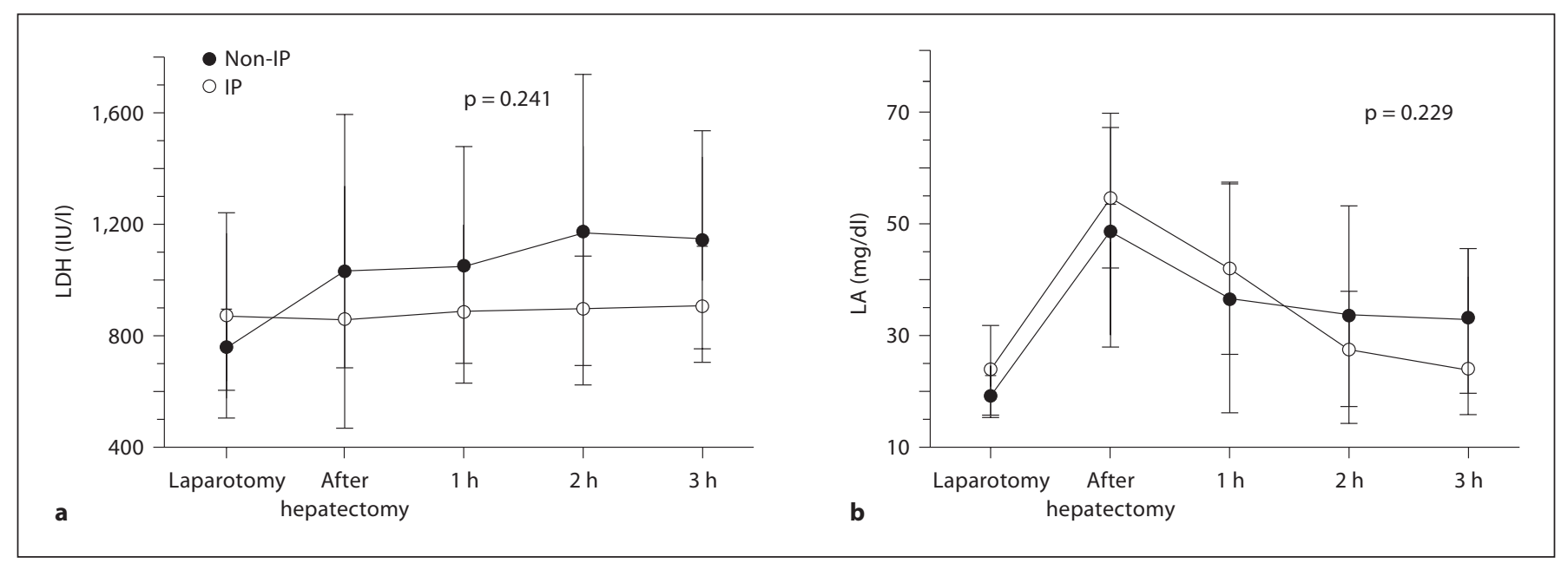

Fig. 3. a There were no significant differences in serum LDH levels between the IP and non-IP group at $3 \mathrm{~h}$. b LA levels in the IP group were lower than those in the non-IP group at 2 and $3 \mathrm{~h}$ after hepatectomy, the intergroup differences were not significant at $3 \mathrm{~h}$.

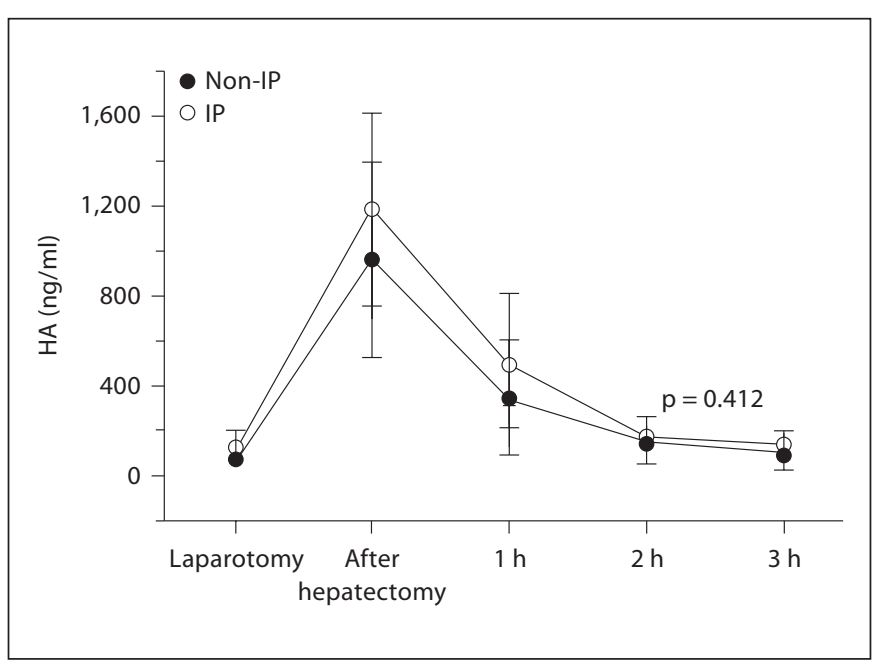

Fig. 4. Immediately after hepatectomy, the serum HA levels of the IP and non-IP group increased and then gradually decreased. However, there were no significant differences in the serum HA levels between the IP and non-IP group at $3 \mathrm{~h}$.

creased gradually after hepatectomy. However, there were no significant differences in serum LDH levels between the 2 groups (fig. 3a). Immediately after hepatectomy, the serum LA levels in both groups elevated and then decreased gradually thereafter. Although the LA levels in the IP group were lower than those in the non-IP group at 120 and $180 \mathrm{~min}$ after hepatectomy, the intergroup differences were not significant (fig. 3b). Immediately after hepatectomy, the serum HA levels in both groups increased and then gradually decreased. However, there were no significant differences in serum HA levels between the groups (fig. 4).

\section{Effect of IP on Plasma TNF- $\alpha$ Levels and $\mathrm{NO}_{2}^{-} / \mathrm{NO}_{3}^{-}$}

The levels of plasma TNF- $\alpha$ in the IP group tended to be lower than those in the non-IP group ( $\mathrm{p}=0.042$; fig. 5a). The $\mathrm{NO}_{2}^{-} / \mathrm{NO}_{3}^{-}$levels in the IP group tended to be lower than those in the non-IP group. However, the differences in plasma $\mathrm{NO}_{2}^{-} / \mathrm{NO}_{3}^{-}$levels between the groups were not significant during the first $180 \mathrm{~min}$ after hepatectomy ( $\mathrm{p}=0.222$; fig. $5 b)$.

\section{Protective Effect of IP against Liver Apoptotic \\ Cell Death}

TUNEL staining at $180 \mathrm{~min}$ after hepatectomy revealed significantly fewer TUNEL-positive cells in the IP group than in the non-IP group (IP group: $31.0 \pm 14.1$ vs. non-IP group: $65.4 \pm 11.5 / 10$ fields; $\mathrm{p}=0.002$; fig. $6 a, b)$.

Electron microscopy revealed apoptosis and necrosis of hepatocytes and sinusoidal endothelial cells in the non-IP group, but such changes were rarely observed in the IP group. In the non-IP group, nuclear chromatin condensation occurred frequently in sinusoidal endothelial cells, and early nuclear chromatin condensation was seen occasionally in hepatocytes. Necrotic hepatocytes showing expanded mitochondria were rarely observed (fig. 7). 
Fig. 5. a Plasma TNF- $\alpha$ levels after hepatectomy for $3 \mathrm{~h}$. The levels in the IP group tended to be lower than those in the nonIP group. ${ }^{*} \mathrm{p}=0.042$. $\mathbf{b}$ The $\mathrm{NO}_{2}^{-} / \mathrm{NO}_{3}^{-}$levels in the IP group tended to be lower than those in the non- IP group. The differences in plasma $\mathrm{NO}_{2}^{-} / \mathrm{NO}_{3}^{-}$levels between the groups were not significant during the first $3 \mathrm{~h}$ after hepatectomy. $\mathrm{p}=0.222$.
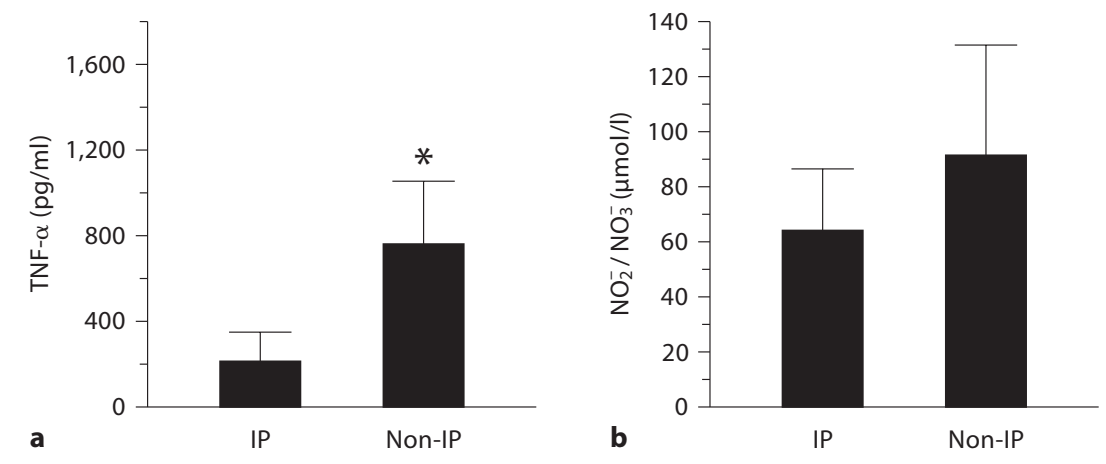

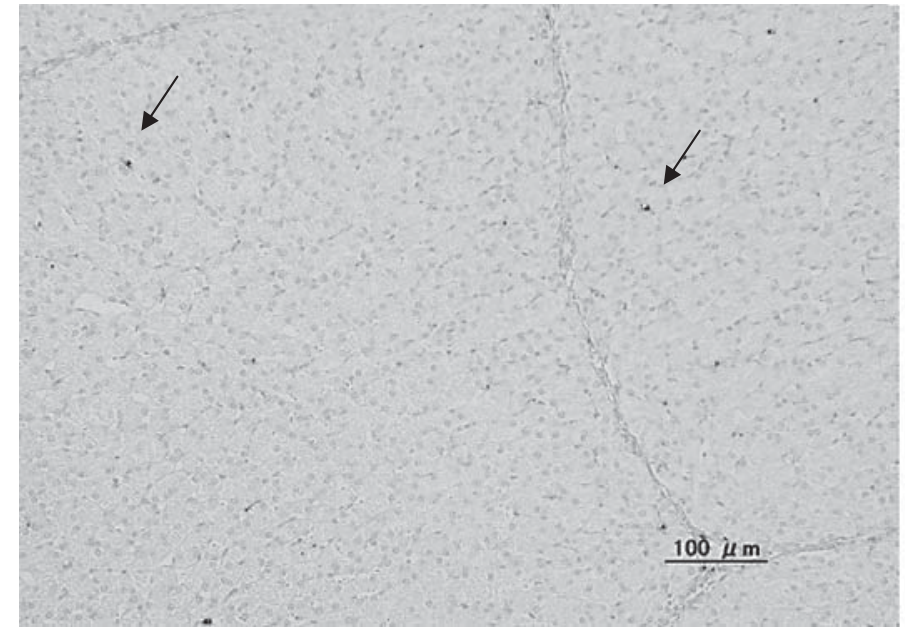

IP

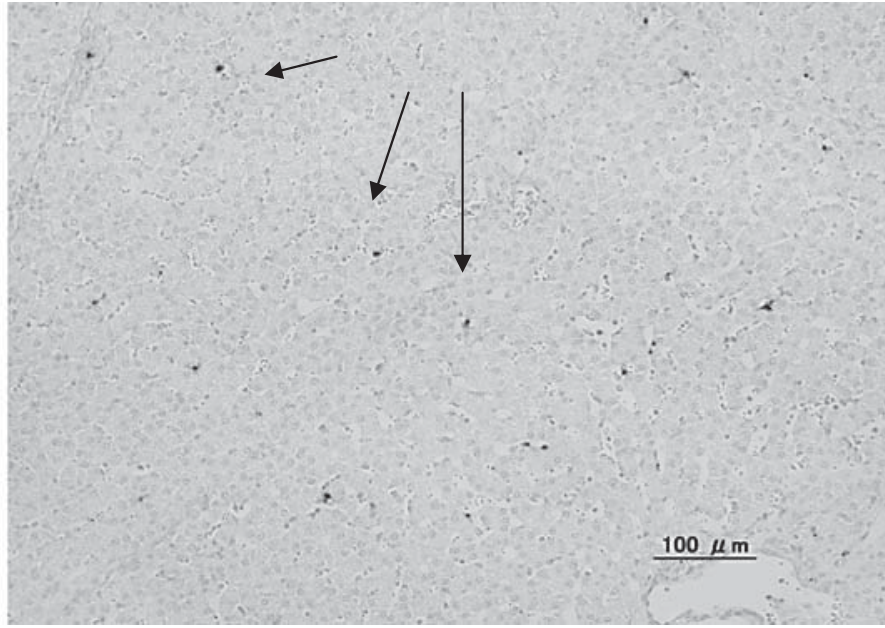

Non-IP

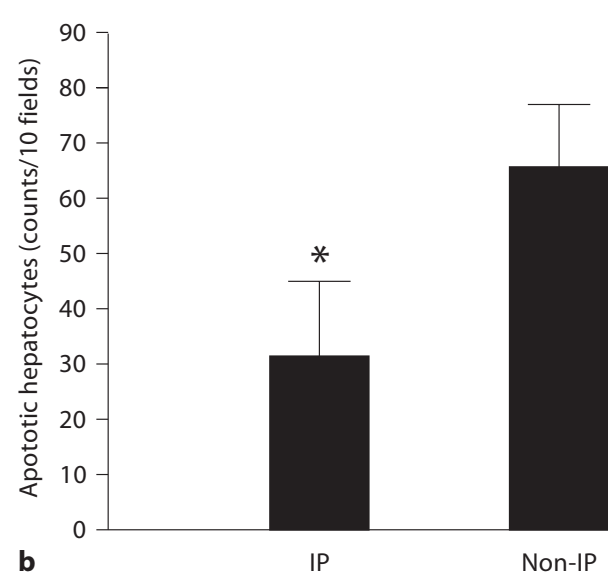

Fig. 6. a TUNEL staining after hepatectomy for $3 \mathrm{~h}$ revealed fewer TUNEL-positive cells (arrows) in the IP than the non-IP group. $\times 200$. b Microphotometric evaluation of TUNEL-positive hepatocytes in TUNEL-stained tissue after reperfusion for $3 \mathrm{~h}$. Values are expressed as means \pm SD. $n=6$ in both groups. The differences between the groups are statistically significant. ${ }^{*} \mathrm{p}=$ 0.002 . 


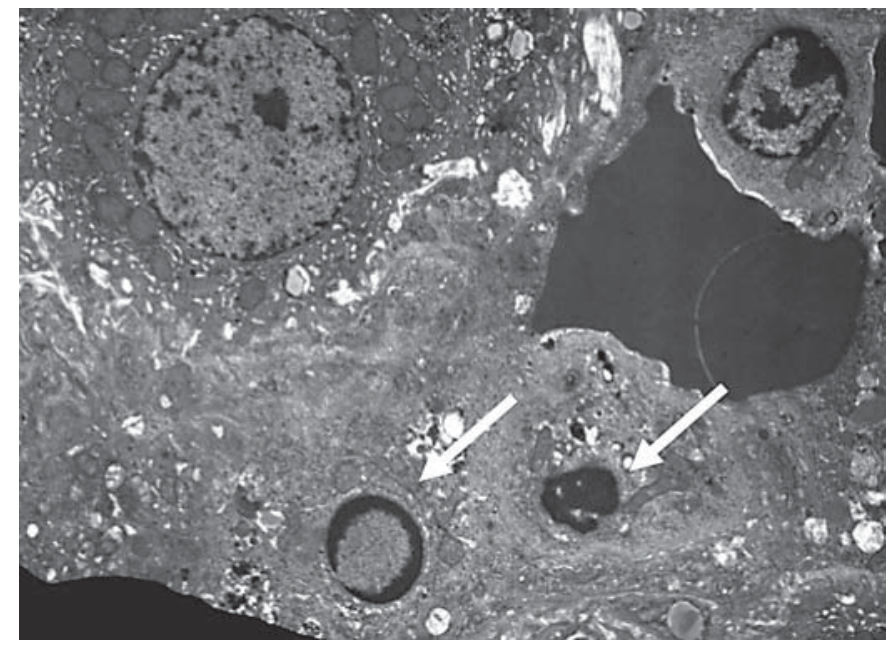

Fig. 7. Electron microscopy revealed apoptotic and necrotic features of hepatocytes and sinusoidal endothelial cells in the non-IP group. Nuclear chromatin condensation occurred frequently in the hepatocytes (arrows).

\section{Discussion}

This is the first experimental study to evaluate the effect of IP plus IC during major hepatectomy in a swine model. IC has been advocated because of its safety during hepatectomy in patients with diseased liver, and it has been suggested that the beneficial effects of IC may be related to the phenomenon of IP [7]. Although the effect of IP has only been reported after continuous hepatic pedicle clamping (CC), its protective effect against liver injury after IC has not yet been discussed. We hypothesized that IP may exert protective effects against liver injury after hepatectomy and conducted a trial using not only CC but also IC.

It is still unclear whether CC or IC is appropriate for liver resection. CC is more effective than IC for decreasing the amount of blood loss during hepatic resection. However, postoperative liver enzyme and bilirubin levels are significantly higher after CC than after IC, especially in patients with liver abnormality [2]. Furthermore, our previous experimental study using a rat model suggested that IC had a better impact on I/R-I than CC [11]. Consequently, IC was defined as repeated clamping for $15 \mathrm{~min}$ followed by reperfusion for $5 \mathrm{~min}$ on the basis of this experimental study. Using this procedure, clinical liver resection has been performed safely at our institution. There is evidence suggesting that hepatic ischemia lasting for more than $15 \mathrm{~min}$ activates calpain proteases [13].
Furthermore, Belghiti et al. [2] have reported a lower rate of complication with IC than with continuous portal triad clamping. On the other hand, the optimal duration of IP is still unclear [14]. Previous studies have suggested that several durations of IP are optimal: 5 min clamping followed by $30 \mathrm{~min}$ reperfusion, 5 or $10 \mathrm{~min}$ of ischemia followed by 10 or $15 \mathrm{~min}$ of reperfusion, or $10 \mathrm{~min}$ of ischemia and $10 \mathrm{~min}$ of reperfusion. In this study, we specified an ischemic period of $10 \mathrm{~min}$ followed by reperfusion for $10 \mathrm{~min}$ before hepatic pedicle clamping [11, 14-19]. This method was found to be the most beneficial after warm ischemia in vivo and prolonged exposure to hypoxia in vitro, and Clavien et al. [15] have reported that 10 min clamping and 10 min reperfusion by IC exerts a protective effect against liver injury in humans [20,21]. Therefore, IP with IC was the most effective procedure protecting for I/R-I.

The serum AST level has been used to evaluate liver damage after liver resection, and is considered to objectively reflect the degree of liver damage [22]. In the present study, serum AST levels were significantly reduced by IP after hepatectomy at $180 \mathrm{~min}$. Levels of other parameters, including serum LA and LDH, tended to be lower in the IP than in the non-IP group. These results indicate that IP protects against liver injury after hepatectomy using IC. As sinusoidal endothelial cells take up HA from blood, the serum HA level has been used to evaluate the function of these cells, and patients with liver cirrhosis or posthepatectomy patients frequently show an increased serum HA level $[23,24]$. In the present study, serum HA levels were similar in both the IP and non-IP groups at each observation point, and IP was effective for protecting sinusoidal endothelial cell function. Although the pathogenesis of liver injury after liver resection using the Pringle maneuver is multifactorial, operative stress such as blood loss and Pringle time play an important role in liver damage during hepatectomy. In our previous study, we found that blood loss and Pringle time did not differ between the 2 groups, and that IP did not affect blood loss or Pringle time. These results suggest that IP promotes liver tolerance through a factor other than reduction of blood loss and Pringle time.

Because of its transient and volatile nature, $\mathrm{NO}$ is difficult to measure directly. However, NO is largely oxidized to $\mathrm{NO}_{2}^{-} / \mathrm{NO}_{3}^{-}$, and the concentration of these anions is often used as a quantitative measure of $\mathrm{NO}$ production. NO acts by maintaining perfusion of the hepatic microcirculation and modulates liver injury through its vasodilatory and anti-inflammatory effects. Experimental studies suggest an association of $\mathrm{NO}$ with 
liver injury in relation to I/R-I. NO has been reported to play a protective role against tissue injury through several mechanisms, including inhibition of leukocyte adhesion to the endothelium, platelet aggregation and a vasodilative action [25]. Several studies have shown that I/R-I is partially mediated by activation of Kupffer cells. Our results also suggest that $\mathrm{NO}_{2}^{-} / \mathrm{NO}_{3}^{-}$was induced to a greater extent in the non-IP group than in the IP group, and that injury to Kupffer cells or microvessels might have induced $\mathrm{NO}_{2}^{-} / \mathrm{NO}_{3}^{-}$. The decreased $\mathrm{NO}_{2}^{-} /$ $\mathrm{NO}_{3}^{-}$levels at $180 \mathrm{~min}$ after reperfusion support the possibility that IP had a protective effect against liver damage.

TNF- $\alpha$ is one of the important extracellular mediators involved in liver damage after hepatic ischemia [26, 27]. Apoptosis is initiated by TNF- $\alpha$ binding to TNF receptor 1 , which activates caspase 8 through the TNF receptorassociated death domain, resulting in induction of DNA fragmentation by the activated caspase cascade [28]. In our study, the serum TNF- $\alpha$ levels at $180 \mathrm{~min}$ after hepatectomy were significantly lower in the IP group than in the non-IP group. Moreover, TUNEL positivity and features of apoptosis such as nuclear chromatin condensation were rarely observed in the IP group with reduced serum TNF- $\alpha$ levels. These results suggest that IP exerts a protective effect by interfering with the increase in serum TNF- $\alpha$, thus preventing apoptotic death.

\section{Conclusion}

We confirmed that IP can exert a protective effect against liver injury not only after liver resection using CC but also after IC. Our study suggested that apoptotic liver damage appeared at an early stage following liver resection using IC, it also confirmed that IP had a protective effect against apoptotic liver damage. Although the protective effect obtained by using IP plus IC is not maximal, our strategy has the potential to improve the clinical postoperative course of patients undergoing liver resection. A clinical study in humans will be required to confirm whether IC followed by IP improves the postoperative condition or not. Thus we have confirmed that liver resection using IP plus IC is considerably superior to the use of IC without IP.

\section{Acknowledgements}

The authors thank Hisato Hirata and Hiromichi Kaneko from the Laboratory Animal Research Center at the Dokkyo University School of Medicine for their excellent technical assistance. This work was supported by a Grant-in-Aid for Scientific Research, KAKENHI (15591435), from the Ministry of Education, Culture, Sports and Technology (MEXT).

\section{References}

1 Pringle JH: Notes on the arrest of hepatic hemorrhage due to trauma. Ann Surg 1908; 48:541-549.

-2 Belghiti J, Noun R, Malafosse R, et al: Continuous versus intermittent portal triad clamping for liver resection: a controlled study. Ann Surg 1999;229:369-375.

-3 Man K, Fan ST, Ng IO, et al: Prospective evaluation of Pringle maneuver in hepatectomy for liver tumors by randomized study. Ann Surg 1997;226:704-711.

4 Belghiti J, Regimbeau JM, Durand F, et al: Resection of hepatocellular carcinoma: a European experience on 328 cases. Hepatogastroenterology 2002;49:41-46.

5 Kubo S, Hirohashi K, Tanaka H, et al: Risk factor for recurrence after resection of hepatitis $\mathrm{C}$ virus-related hepatocellular carcinoma. World J Surg 2000;24:1559-1565.

6 Kubo S, Hirohashi K, Yamazaki O, et al: Effect of the presence of hepatitis B e antigen on prognosis after liver resection for hepatocellular carcinoma in patients with chronic hepatitis B. World J Surg 2002;26:555-560.
7 Ikai I, Kojiro M, Ichida T, et al: Reevaluation of prognostic factors for survival after liver resection in patients with hepatocellular carcinoma in a Japanese nationwide survey. Cancer 2004;101:796-802.

8 Makuuchi M, Mori T, Gunven P, et al: Safety of hemihepatic vascular occlusion during resection of the liver. Surg Gynecol Obstet 1987;164:155-158.

$\checkmark 9$ Horiuchi T, Muraoka R, Tabo T, et al: Optimal cycles of hepatic ischemia and reperfusion for intermittent pedicle clamping during liver surgery. Arch Surg 1995;130:754758.

$>10$ Murry CE, Jennings RB, Reimer KA: Preconditioning with ischemia: a delay of lethal cell injury in ischemic myocardium. Circulation 1986;74:1124-1136.

11 Iwasaki Y, Tagaya N, Hattori Y, et al: Protective effect of ischemic preconditioning against intermittent warm-ischemia-induced liver injury. J Surg Res 2002;107:8292.
12 Van Wagensveld BA, Van Gulik TM, Gelderblom HC, Scheepers JJ, Bosma A, Endert E, Gouma DJ: Prolonged continuous or intermittent vascular inflow occlusion during hemihepatectomy in pigs. Ann Surg 1999; 229:376-384.

13 Kohli V, Madden JF, Bentley RC, et al: Calpain mediates ischemic injury of the liver through modulation of apoptosis and necrosis. Gastroenterology 1999;116:168-178.

14 Clavien PA, Selzner M, Rudiger HA, et al: A prospective study in 100 consecutive patients undergoing major liver resection with versus without ischemic preconditioning. Ann Surg 2003;238:843-852.

15 Clavien PA, Yadav S, Sindram D, et al: Protective effects of ischemic preconditioning for liver resection performed under inflow occlusion in humans. Ann Surg 2000;232: 155-162.

16 Eipel C, Glanemann M, Nuessler AK, et al: Ischemic preconditioning impairs liver regeneration in extended reduceed-size livers. Ann Surg 2005;241:477-484. 
17 Koti, RS, Seifalian AM, Davidson BR, et al: Protection of the liver by ischemic preconditioning: a review of mechanism and clinical applications. Dig Surg 2003;20:383-339.

18 Glanemann M, Strenziok R, Kuntze R, et al: Ischemic preconditioning and methylprednisolone both equally reduce hepatic ischemia/reperfusion injury. Surgery 2004;135: 203-214.

19 Yadav SS, Sindram D, Pretty DK, et al: Ischemic preconditioning protects the liver by inhibition of apoptosis through a caspasedependent pathway. Hepatology 1999;30: 1223-1231.

20 Peralta C, Closa D, Xaus C, et al: Hepatic preconditioning in rats is defined by a balance of adenosine and xanthine. Hepatology 1998; 28:768-773.
21 Carini R, Cesaris MGD, Splendore R, et al: Ischemic preconditioning reduces $\mathrm{Na}^{+}$accumulation and cell killing in isolated rat hepatocytes exposed to hypoxia. Hepatology 2000;31:166-172.

22 Iu S, Harvey PRC, Makowka L, et al: Markers of allograft viability in the rat. Transplantation 1987;44:562-569.

23 Deaciuc IV, Bagby GL, Lang CH, et al: Hyaluronic acid uptake by the isolated, perfused rat liver: an index of hepatic sinusoidal endothelial cell function. Hepatology 1993; 17: 266-272.

24 Smedsrod B, Pertoft H, Eriksson S, et al: Studies in vitro on uptake and degradation of sodium hyaluronate in rat liver endothelial cells. Biochem J 1984;223:617-626.
25 Ma TT, Ischiropoulos H, Brass CA: Endotoxin-stimulated nitric oxide production increases injury and reduces rat liver chemiluminescence during reperfusion. Gastroenterology 1995; 108:463-469.

26 Colletti LM, Remick DG, Burtch GD, et al: Role of tumor necrosis factor- $\alpha$ in the pathophysiologic alterations after hepatic ischemia/reperfusion injury in the rat. J Clin Invest 1990;85:1936-1943.

27 Kimura N, Muraoka R, Horiuchi T, et al: Intermittent hepatic pedicle clamping reduces liver and lung injury. J Surg Res 1998;78:1117.

28 Rath PC, Aggarwal BB: TNF-induced signaling in apoptosis. J Clin Immunol 1999;19: 350 . 